\title{
ERRATUM
}

Weihong Yang · Keiichi Watanuki - Shen Zhao

\section{A quick intelligent control system for a mobile robot to avoid collision with moving obstacles}

Published online: 25 October 2005

(C) Springer-Verlag 2005

\section{Microsyst Technol (2005) 11:569-576}

Due to an unfortunate error, Eq. 9 was published incorrectly. The corrected version is shown below:

$\left|\mathbf{P}_{\mathrm{o} 2} \mathbf{P}\right|=\frac{\left|\mathbf{P}_{\mathrm{r}} \mathbf{P}_{\mathrm{o}}(0)\right| \cdot \sin \theta_{\mathrm{ro} 2}}{\sin \beta}+\frac{\varepsilon_{1}}{\sin \beta}+L_{o}-\frac{\varepsilon_{2}}{\tan \beta}$
In the unnumbered equation between Eqs. 12 and 13

$>$ should be $<$, see below:

$\sin \left(\beta-\theta_{\text {ro } 2}\right)<\frac{\left|\mathbf{V}_{\mathbf{r}}(0)\right|}{\left|\mathbf{V}_{\mathbf{0}}(0)\right|} \sin \theta_{\text {ro2 },}$
The online version of the original article can be found http:// dx.doi.org/10.1007/s00542-005-0556-y

W. Yang $(\bowtie) \cdot$ K. Watanuki

Department of Mechanical Engineering,

Faculty of Engineering, Saitama University,

255 Shimo-Okubo, Sakura-Ku, Saitama-Shi,

Saitama 338-8570, Japan

E-mail: yang@mech.saitama-u.ac.jp; yangwh5@hotmail.com

Tel.: + 81-48-8589576

Fax: + 81-48-8562577

S. Zhao

Omori Unit, Minebea Co., Ltd.

4-18-18 Omori-Nishi, Ota-Ku,

Tokyo 143-8543, Japan 